\title{
PIQL Preservation Services - A Holistic Approach to Digital Long-term Preservation
}

\author{
Alexander Rych \\ In-Vision Digital Imaging Optics GmbH \\ Industriestrasse 9, Guntramsdorf, Austria \\ rych@piql.at
}

\section{Summary}

Digital storage media (hard disks, magnetic tape) exhibit limited lifetime. Repetitive data migration to overcome rapid obsolescence of hardware and software bears accelerated risk of data loss, data corruption or even manipulation and adds significant repetitive costs for hardware and software investments.

So Piql Preservation Services ("Piql") has been developed as a turnkey solution designed for secure, migration-free long-term preservation of digital data. It sets an open standard for long-term preservation for the future, consisting of equipment and processes needed for writing and retrieving digital data.

Keywords: Piql, long-term preservation, archive, digital data on analogue media, film, digital frames, hybrid preservation

\section{PIQL - Solution}

Piql stores any kind of data in its digital as well as analog form securely on a film reel for 500 years. Using photosensitive film polyester base, a very stable material that is known for its immutability over hundreds of years, secure and cost-effective long- term preservation can be provided. The film reel itself is stored in a packaging capable of protecting the optical storage medium. These components have undergone extensive testing to ensure longevity of up to 500 years. In addition to its durability, film is a true WORM (write once-read many) medium. It therefore is resistant to editing or manipulation.

Being able to store any form of data onto the film makes Piql a superior solution for long-term preservation. Paper documents, images, video or audio sequences - all of those file formats and documents can be preserved in its native file structure. In order to restore the encoded digital data, only a film scanner, a digital camera or any appropriate optical reading device will be needed in the future. Every film reel includes besides meta-data all necessary information to enable users in the future to read and decode the digital information. 
Key features:

- Migration-Free. By storing digital data in archival file formats on the unique piqlFilm, repetitive migrations are no longer needed. Users avoid the risk of migration-related corruption and data loss and thus save longterm costs.

- Searchable. Operated within a standard IT-environment, the user's data is indexed and fully searchable - today and in the future.

- Flexible. Depending on the user's needs, data can either be stored as a human- readable text or image or in a digital format, making dlata readable and understandable for 500 years.

- Unalterable. Data is preserved on a secure true WORM medium, making it impossible to manipulate or delete valuable data.

- Permanent. Photosensitive film is known to be robust with proven longterm qualities. PiqlFilm is tested to last for 500 years. Although your time perspective may be shorter, you can be assured that your data will remain intact.

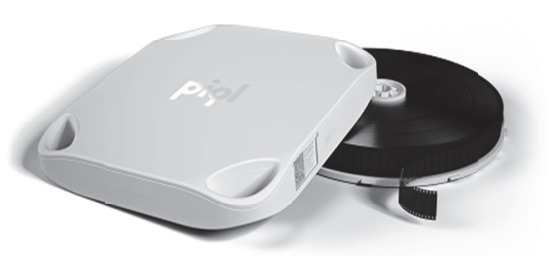

Figure 1: piqlBox and piqlFilm

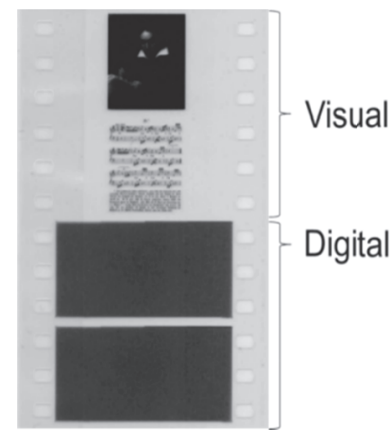

Fig. 2: digital and visual representation of data

The workflow consists in data boxing and integrity check, data recording, data scanning to guarantee integrity and accessibility in the future - storage and data unboxing (process that fully restores the scanned data). 
Piql Preservation Services manage to combine some of the most important components to ensure a secure and cost-effective long-term archiving system.

- There is no limit of what can be stored onto the piqlFilm. Digital data such as HD audio-visual data, documents or databases can be archived with Piql.

- It is a service that uses a whole new approach to long-term preservation by removing the need for constant data migration, reducing hardware and software maintenance.

- The innovation is to use a permanent media, to adopt standards for formats and interfaces and be independent of proprietary software and specific hardware.

Piql Preservation Services convert digital files into a physical copy that can be stored as the ultimate digital insurance. Data owners can feel confident about the long-term accessibility of their valuable data - today and in 500 years. 